ORIGINAL ARTICLE

\title{
Performance of routine syphilis serology in the Ethiopian cohort on HIV/AIDS
}

\author{
J W Dorigo-Zetsma, D Belewu, H Meless, E Sanders, R A Coutinho, A Schaap, D Wolday
}

Sex Transm Infect 2004;80:96-99. doi: 10.1136/sti.2003.005827

\begin{abstract}
See end of article for authors' affiliations

.....................

Correspondence to: J W Dorigo-Zetsma, $\mathrm{MD}, \mathrm{PhD}$,

Ethio-Netherlands AIDS

Research Project,

Ethiopian Health and

Nutrition Research

Institute, PO Pox 1242,

Addis Ábaba, Ethiopia

moud@gggd.

amsterdam.nl
\end{abstract}

Accepted for publication 22 September 2003

\begin{abstract}
Objectives: To assess the performance of routine syphilis screening during 5 year follow up of Ethiopian factory workers, participating in a cohort study on HIV/AIDS.

Methods: Syphilis serology test results of factory workers, who each donated at least six blood samples were evaluated. Screening in 1997-8 had been performed by the Treponema pallidum particle agglutination (TPPA) assay and in 1999-2001 by the rapid plasma reagin (RPR) test. TPPA had been followed by RPR or RPR by TPPA, in case of a positive screening result. Samples of study subjects showing inconsistent sequential TPPA and/or RPR results were retested independently by three laboratory technicians.

Results: A total of 540 cohort participants (8.3\% HIV positive at enrolment) donated 4376 blood samples (mean 8.3 per subject). From 93 of the 176 participants with at least one positive TPPA result during follow up, 152 samples were retested by RPR and/or TPPA. Based on the revised syphilis test results, the 540 cohort participants were classified as having no $(70.5 \%)$, past $(20.6 \%)$, prevalent $(6.9 \%)$, or incident $(2.0 \%)$ syphilis. The RPR screening test was difficult to interpret and yielded $8.2 \%$ biological false positive (BFP) RPR results, or $3.2 \%$ if weak positive results were excluded. There was no correlation between HIV infection and BFP RPR reactions. Sample mix-ups were detected in 1.2\%.

Conclusion: Evaluation of routine syphilis screening as performed in a long term cohort study on HIV/AIDS in Ethiopia showed difficulties encountered in syphilis screening programmes such as a high percentage of BFP RPR, inconsistencies in interpretation of the RPR test, and sample mix ups. The findings stress the need to develop a syphilis screening assay that is easy to perform and interpret and to implement quality assurance programmes.
\end{abstract}

n many developing countries syphilis remains an important cause of morbidity and is associated, like other ulcerative sexual transmitted infections, with enhanced sexual transmission of HIV. ${ }^{1}$ Figures on prevalence and incidence of syphilis in several studies from sub-Saharan Africa are based on commonly used serological screening assays. ${ }^{2-6}$ However, the interpretation of non-treponemal and treponemal specific serological tests in a population where syphilis and HIV are endemic, is encountered with difficulty..$^{7-9}$

The rapid plasma reagin (RPR) test is a non-treponemal serological test for syphilis widely used as a screening test in antenatal clinics and other health facilities in the developing world. The test is easy to perform, does not need advanced equipment, and is inexpensive. However, the RPR test may be difficult to interpret, it has shown both biological false positive (BFP) and false negative results and, particularly in HIV infected patients, the test has been known to generate BFP results. ${ }^{7810}$ The limited specificity of the test can be ascribed to the aspecific nature of the cardiolipin antigen. In contrast, treponemal antibody tests such as the Treponema pallidum particle agglutination (TPPA) test and the fluorescent treponemal antibody (FTA) absorption test have a high specificity, but in HIV infected patients false negative reactions have been reported. ${ }^{911}$

Data on the outcome of routine syphilis serological diagnostics during a long term follow up period in developing countries are scarce. Evaluation of such data will give valuable information on how to interpret syphilis diagnostic test results in a population where HIV is also common. The objectives of this study therefore were to evaluate the performance of routine syphilis serodiagnosis among participants with a minimum of six follow up visits in the cohort studies on HIV/AIDS in Ethiopia (ENARP study) and provide data on prevalence and incidence of syphilis in this population

\section{PATIENTS AND METHODS \\ ENARP cohort}

The ongoing cohort study on HIV-1 infection incidence and progression was started in a factory in the suburbs of Addis Ababa, Ethiopia, in February 1997. Factory workers aged 1844 years were enrolled, and participants gave consent to regular serological testing for both HIV and syphilis during follow up visits every 6 months. At each visit blood is drawn using EDTA vacutainer tubes (Becton and Dickinson, USA). Plasma is separated and tested for antibodies against HIV-1 by enzyme linked immunosorbent assay (ELISA, Vironostika HIV Uni-form II Plus O, Organon Teknika, Boxtel, Netherlands), with results confirmed by western blot (Genelabs Diagnostics Inc, Redwood City, CA, USA). Until July 1999, screening for syphilis was performed with a TPPA test (Serodia-TP, Fujirebio Inc, Tokyo, Japan), and in case of a positive TPPA result, the sample was tested for anticardiolipin antibodies with an RPR test (RPR Slide test, Biomerieux, Marcy l'Etoile, France). Since July 1999, because of the high percentage of TPPA positives in the study population, screening has been performed with RPR (RPRNosticon II, Organon Teknika, Boxtel, Netherlands) and followed by TPPA in case of a positive RPR. Tests are performed and interpreted according to instructions of the manufacturers. Plasma samples for RPR are tested undiluted, plasma samples for TPPA are tested in a 1:80 dilution.

Until July 1999, participants with positive TPPA on cohort enrolment were offered treatment for latent syphilis, three doses each of 2.4 million units of benzathine penicillin weekly, since reliable history of previous treatment is difficult 
to obtain. Sexual partners were also offered free treatment. Participants enrolled since July 1999 have been offered treatment for active syphilis (2.4 million units of benzathine penicillin, single dose) when a sample tested positive for both RPR and TPPA.

Blood samples are received daily at the ENARP laboratory in Addis Ababa, where they are registered. Within 1 week of arrival, plasma samples are tested using standard operating procedures, and test results are entered in the ENARP database. The laboratory participates in the external quality control programme for syphilis serology conducted biannually by the CDC, Atlanta, USA, with a score of $>95 \%$ for RPR and $100 \%$ for TPPA during the past 5 years. After testing, plasma samples are stored at $-80^{\circ} \mathrm{C}$ with a unique laboratory identification number to ensure efficient retrieval.

\section{Database screening, sample selection for retesting, and syphilis serodiagnosis}

For our study, we used the ENARP database to identify participants with six or more visits between their enrolment and December 2001. All eligible participants had been enrolled before July 1999, so at least one TPPA result was available for each of them. Based on syphilis results at their enrolment visit and follow up visits, participants were divided into three categories:

(a) no syphilis: syphilis tests on all visits negative

(b) biological false positive (BFP) syphilis tests: one or more weak positive or positive RPR tests, but negative TPPA

(c) at least one positive TPPA result, with or without positive RPR result.

Participants in categories (a) and (b) were interpreted as having no syphilis. For about half of the participants in category (c), it was difficult to interpret sequential syphilis test results because of inconsistencies. To establish a syphilis serodiagnosis for these participants, samples showing inconsistent sequential syphilis test results were retested in RPR (RPR-Nosticon II, Organon Teknika, Boxtel, Netherlands) and/or TPPA (Serodia-TP, Fujirebio Inc, Tokyo, Japan). Criteria for samples to be retested were a positive TPPA result among several negative TPPA results, or vice versa, a negative RPR result among several positive RPR results (with positive TPPA results), or a positive RPR result (with positive TPPA result) among several negative RPR results. Three laboratory technicians who were blinded to the previous results performed testing independently. Weak reactive RPR tests were interpreted as positive. A consensus test result was obtained if all three technicians reported the same findings. Consensus results were compared with the previously obtained results and were subsequently entered in the database. In case the three disagreed, the result from two technicians reporting the same result was entered.

Using the revised syphilis results, subjects in category (c) were classified as having past, prevalent, incident or no syphilis. Since these definitions were based on serology only, rather than combined serological and clinical findings, we did not use the standard terminology of primary, secondary, early, or late latent syphilis. ${ }^{12}$ Definitions were as follows: past syphilis positive TPPA since enrolment without positive RPR; prevalent syphilis positive RPR and TPPA results since enrolment; incident syphilis sequential RPR seroconversion with or without TPPA seroconversion (in case TPPA was positive throughout), and no syphilis tests on all visits negative. The incidence of syphilis was calculated per 100 person years of follow up, and confidence intervals were derived assuming a Poisson distribution of events. Cohort participants were divided according to their syphilis serostatus into age groups based on age at enrolment. The $\chi^{2}$ test for trend with one degree of freedom was used to test increasing prevalence of past, prevalent, and incident syphilis infection with age.

For all samples with a positive TPPA screening result from enrolment until July 1999, the percentage of sample mix-ups was calculated, using the fact that TPPA remains positive for a long period, if not lifelong.

For all samples screened with RPR from July 1999 onwards, the percentage of BFP RPR test results was calculated.

Association between HIV seropositivity and BFP RPR test results was tested by comparing HIV prevalence at enrolment among participants with BFP RPR test results and those with no serological evidence of syphilis using Pearson $\chi^{2}$ test. Association between HIV seropositivity and syphilis was tested by comparing HIV prevalence at enrolment among participants with incident, prevalent, or past syphilis and those with no serological evidence of syphilis using Pearson $\chi^{2}$ test. A $p$ value of $<0.05$ was considered significant. Data were analysed using the Stata statistical package version 6.0 (Stata Statistical Software, Stata Corporation, College Station, TX, USA).

\section{RESULTS}

Out of 824 enrolled factory workers, 540 (66\%) who had six or more cohort visits between February 1997 and December 2001, were selected for this study. Their mean age at enrolment was 35 years; $54 \%$ were males, $8.3 \%$ were HIV positive at enrolment, and $11.3 \%$ HIV positive at December 2001. They donated 4376 blood samples (mean 8.3 per subject). Table 1 shows how subjects were divided into categories (a), (b), and (c) according to their syphilis serological test results.

Category (c) included 176 subjects with at least one positive TPPA result during follow up. For 93 subjects (53\%) in this category it was impossible to establish a syphilis serodiagnosis because of inconsistent sequential syphilis test results. A total of 152 samples from the 93 participants were selected for retesting by TPPA $(n=18)$, by RPR $(n=100)$, or by both tests $(\mathrm{n}=34)$. Consensus, with all three technicians reporting the same result, was reached for TPPA in all 52 samples (100\%). For RPR, the three technicians agreed in 99 of 134 samples $(74 \%)$. In table 2 the performance of each technician is shown, with a positive RPR result being the least subject to inter-individual variation in interpretation.

After retesting, 17 subjects turned out to be completely negative for syphilis. Based on the revised results, the 540 cohort participants could be classified as having no (70.5\%), past $(20.6 \%)$, prevalent $(6.9 \%)$, or incident $(2.0 \%)$ syphilis (table 1). Syphilis incidence in the cohort was calculated to be 0.5 (95\% CI: 0.2 to 0.7 ) per 100 person years.

From enrolment until July 1999, 785 samples were sequentially screened for TPPA and had a positive TPPA result. Among these, nine (1.2\%) were definite sample mixups, being (confirmed) TPPA negative, among several positive TPPA results.

From July 1999 until December 2001, 2325 samples were sequentially screened for RPR. Among these, the percentage biological false positive RPR tests including both weak positive RPR $(\mathrm{n}=115)$ and positive RPR samples $(\mathrm{n}=75)$ was $8.2 \%$ (190/2325). Excluding the weak positive RPR samples, the false positive rate was $3.2 \%$.

False positive RPR reactions were equally divided among the 170 participants in category (b), and no subject with chronic false positive reactions was seen. HIV seropositivity was not correlated with false positive RPR results, with 11 / $170(6.5 \%)$ of such participants being HIV positive compared with $14 / 211(6.6 \%)$ participants with no evidence of syphilis $(\mathrm{p}=0.95)$. Excluding participants with weak positive RPR results there was still no correlation, with 6/70 (8.6\%) participants with false positive RPR results being HIV positive 
Table 1 Interpretation of sequential testing for syphilis among 540 Ethiopian factory workers who donated a mean of eight consecutive blood samples during 5 years of follow up, before and after retesting for sequential inconsistent RPR/TPPA results

\begin{tabular}{|c|c|c|c|c|c|}
\hline & \multicolumn{5}{|c|}{ No of subjects categorised according to their test results before retesting } \\
\hline & Category (a) & Category (b) & Category (c) & & \\
\hline \multirow{3}{*}{$\begin{array}{l}\text { Interpretation of test results in } \\
\text { subjects after retesting of } \\
\text { samples in } 93 \text { subjects in (c) }\end{array}$} & $\begin{array}{l}194 \text { subjects with all } \\
\text { consecutive samples RPR-/ } \\
\text { TPPA- }\end{array}$ & $\begin{array}{l}170 \text { subjects with at least } \\
\text { one sample RPR+/TPPA- }\end{array}$ & $\begin{array}{l}176 \text { subjects with at } \\
\text { least one sample } \\
\text { TPPA+ }\end{array}$ & & \\
\hline & $\begin{array}{l}\text { No syphilis (consecutive } \\
\text { samples TPPA-) }\end{array}$ & & $\begin{array}{l}\text { Past syphilis } \\
\text { (consecutive samples } \\
\text { RPR-/TPPA+) }\end{array}$ & $\begin{array}{l}\text { Prevalent syphilis } \\
\text { (consecutive samples } \\
\text { RPR+/TPPA+) }\end{array}$ & Incident syphilis $\dagger$ \\
\hline & $364+17^{*}$ subjects $(70.5 \%)$ & & 111 subjects (20.6\%) & 37 subjects $(6.9 \%)$ & 11 subjects (2.0\%) \\
\hline
\end{tabular}

*17 subjects, formerly classified as (c), were interpreted as no syphilis after retesting.

tDefined as consistent sequential RPR seroconversion with or without (in case TPPA was positive throughout) TPPA seroconversion.

compared with $14 / 211(6.6 \%)$ participants with no syphilis $(\mathrm{p}=0.59)$.

Table 3 shows the age distribution related to syphilis serostatus. The prevalence of incident/prevalent/past syphilis increased with age (test for trend, $\mathrm{p}<0.001$ ).

HIV seropositivity was highly associated with serological evidence of syphilis, whether incident, prevalent, or past syphilis, with $28 / 159$ (17.6\%) of such participants being HIV positive compared with $33 / 381(8.7 \%)$ participants with no evidence of syphilis $(\mathrm{p}=0.003)$.

Among the 11 seroincident syphilis participants, two were HIV positive at enrolment and two seroconverted for HIV during the study. In one case, syphilis seroconversion and HIV seroconversion were detected in the same sample; in the other case, syphilis seroconversion was detected 6 months before HIV seroconversion.

\section{DISCUSSION}

Treponemal antigen based enzyme immunoassays (EIAs) have recently been introduced as an appropriate alternative for syphilis screening, ${ }^{13}$ and are reported to be highly sensitive and specific compared to the commonly used RPR/ TPPA screening..$^{14}$ In developing countries, however, where ELISA technology is not readily available in most places, the use of cardiolipin antigen based RPR tests combined with Treponema pallidum agglutination (TPPA) tests is still standard, ${ }^{15}$ and many facilities have only RPR or Veneral Disease Reference Laboratory (VDRL) tests. When positive findings can not be confirmed in treponemal specific antibody tests, ${ }^{16}$ overtreatment of syphilis may result because of BFP reactions in the cardiolipin antigen tests. In the present study, routine screening for syphilis was performed using both RPR and TPPA assays. With RPR, the BFP rate was as high as $8.2 \%$ but dropped to $3.2 \%$ if weak positive results were considered negative. This figure is higher than found in the general populations in Zimbabwe and Jamaica ${ }^{11}{ }^{17}$ but agrees with findings in a recent study from rural communities in the Gambia. ${ }^{16}$ In the Gambian study, West et al calculated positive predictive values of the RPR test in relation to the prevalence of active syphilis in the community. ${ }^{16}$ Their findings suggest that in our setting, with a prevalence of active syphilis of $6.9 \%$, the chance of someone with a positive test having active syphilis is less than $60 \%$, and some overdiagnosis and overtreatment is bound to occur.

Although in some studies more BFP RPR results among HIV positive patients were reported, ${ }^{9}{ }^{10}$ we did not find such correlation. However, populations in these studies (injecting drug users ${ }^{9}$ and STD clinic attendee ${ }^{10}$ ) are not comparable with our healthy cohort participants, and as BFP RPR results are influenced by population characteristics, ${ }^{8}$ performance of the RPR test should be studied in the population where it is being used. Not all false positive RPR reactions in our study may be the result of biological factors, but it is possible also to be the result of limitations of the RPR test itself and subjective interpretation. This holds true particularly for the RPR reactions reported as weakly positive. However, upon excluding weak positive results, there was still no correlation between false positive RPR and HIV positivity. The BFP RPR reactions in our study population were all of transient nature and thus are more likely to be associated with acute intercurrent infections, ${ }^{8}$ such as relapsing fever, which is quite common in Ethiopia. The observation that BFP RPR reactions in HIV positive people might represent, in fact, false negative treponemal serology ${ }^{11}$ could also not be confirmed in our population, since these reactions were seen in one visit only. They occurred equally often in HIV positive and HIV

Table 2 Result of RPR tests in 134 selected samples independently retested by three laboratory technicians

\begin{tabular}{lllll}
\hline RPR result & Technician 1 & Technician 2 & Technician 3 & Consensus (3 technicians reporting same result) \\
\hline Negative & 60 & 58 & 54 & 42 \\
Weak positive & 26 & 27 & 25 & 12 \\
Positive & 48 & 49 & 55 & 45 \\
Total & 134 & 134 & 134 & 99 (74\%) \\
\hline
\end{tabular}

Table 3 Age distribution among study subjects according to syphilis serostatus

\begin{tabular}{llllll}
\hline Age & Incident (\%) & Prevalent (\%) & Past (\%) & No infection (\%) & Total \\
\hline$<25$ & $0(0)$ & $1(7.1)$ & $0(0)$ & $13(92.9)$ & 14 \\
$25-34$ & $5(2.5)$ & $11(5.6)$ & $25(12.8)$ & $155(79.1)$ & 196 \\
$35-44$ & $6(1.9)$ & $22(7.1)$ & $80(26)$ & $200(64.9)$ & 308 \\
$>44$ & $0(0)$ & $3(13.6)$ & $6(27.3)$ & $13(59.1)$ & 22 \\
Total & $11(2)$ & $37(6.8)$ & $111(20.6)$ & $381(70.6)$ & 540 \\
\hline
\end{tabular}




\section{Key messages}

- Routine screening for syphilis in developing countries encounters difficulties such as a high percentage of (biological) false positive RPR tests

- (External) quality control in such screening programmes is essential to validate RPR testing results and reduce sample mix-ups

- In our setting there was no correlation between HIV infection and biological false positive RPR reactions

- Although prevalence of past/current syphilis was high in the population under study, the incidence was low

negative people, and none of them seroconverted for treponemal specific antibodies during follow up visits.

This study was based on syphilis serological test results obtained with RPR and TPPA, both tests having limitations as epidemiological tools. ${ }^{18}$ However, our longitudinal cohort was screened for syphilis every 6 months, giving us the unique opportunity to interpret syphilis serology over time. Retesting of samples from participants with inconsistent syphilis results obtained during follow up was necessary to enable proper interpretation. Apart from inter-individual variations in interpretation of the RPR test, retesting revealed sample mix-ups in $1.2 \%$. Reasons for sample mix-ups are difficult to retrieve, but at least in our setting handwritten labels and worksheets in the laboratory have been one reason, and handwriting was replaced by printed labels and electronically generated worksheets. Although usually not a major problem in epidemiological data analysis, sample mix-ups definitely do occur in clinical and laboratory practices, ${ }^{19} 20$ suggesting that quality assurance systems should be implemented to ensure reliable data for analysis.

Among our population in Ethiopia, we found a prevalence of past/prevalent/incident syphilis of $29.5 \%$, which is high compared to prevalences found in other African studies, whether in rural $^{26}$ or urban ${ }^{41}$ populations. As expected, because of the presumed lifelong seropositivity of TPPA, the prevalence of past syphilis increased with age. Incident cases were defined as both TPPA seroconverters and RPR seroconverters, although the latter were already positive in TPPA. This was done to capture new episodes of syphilis among those previously infected. Using this definition, 11 seroincident cases were found, among whom four with a new infection and seven with a re-infection. This is a higher number than previously reported in the same cohort, ${ }^{3}$ but in the same range as seroconverters for HIV in the population under study. ${ }^{21}$ In terms of prevalence there was a strong correlation between syphilis and HIV, but only four of the incident syphilis cases were or became HIV infected. Seroincident cases were equally spread among males and females, with a mean age of 36.1 and 34.6 years respectively, contradicting findings that syphilis affects primarily older men. ${ }^{22}$

This study revealed a high percentage of BFP RPR results, inconsistent inter-individual interpretation of the nontreponemal RPR test, and sample mix ups. In populations like ours, the use of the RPR test as a single screening assay for syphilis is questionable. However, although both syphilis and HIV are common in this population, there was no indication that syphilis serological tests were influenced by HIV infection.

\section{ACKNOWLEDGEMENTS}

We thank A Beyene, D Tefera, and A Tafesse for technical assistance, NHTM Dukers and JPF Schellekens for comments on the manuscript, and LD Phillips for editorial review.
The Ethio-Netherlands AIDS Research Project (ENARP) is a collaborative effort of the Ethiopian Health and Nutrition Research Institute (EHNRI), Addis Ababa, the Municipal Health Service, Amsterdam, the Department of Human Retrovirology of the Academic Medical Centre (University of Amsterdam), and the Central Laboratory of the Netherlands Red Cross Blood Transfusion Service. ENARP is financially supported by the Dutch Ministry for Development Co-operation and the Ethiopian Ministry of Health.

\section{CONTRIBUTORS}

JWDZ designed the study, coordinated the laboratory testing, and drafted the report; DB coordinated the fieldwork and syphilis treatment; HM supervised and contributed to the laboratory testing; ES and RC contributed to the study design and editing of the report; AS was responsible for data handling and analysis; DW contributed to the study design, coordination of laboratory testing, and edited the report.

\section{Authors' affiliations}

J W Dorigo-Zetsma, D Belewu, H Meless, E Sanders, A Schaap,

D Wolday, Ethio Netherlands AIDS Research Project (ENARP), Ethiopian Health and Nutrition Research Institute (EHNRI), Addis Ababa, Ethiopia R A Coutinho, Division of Public Health and Environment, Municipal Health Service, Amsterdam, Netherlands

\section{REFERENCES}

1 Cohen MS. Sexually transmitted diseases enhance HIV transmission: no longer a hypothesis. Lancet 1998;351(Suppl):5-7.

2 Todd J, Munguti K, Grosskurth H, et al. Risk factors for active syphilis and TPHA seroconversion in a rural African population. Sex Transm Infect $2001 ; 77: 37-45$.

3 Sahlu T, de Wit TR, Tsegaye A, et al. Low incidence of syphilis among factory workers in Ethiopia: effect of an intervention based on education and counselling. Sex Transm Infect 2002;78:123-6.

4 Bakari M, Lyamuya E, Mugusi F, et al. The prevalence and incidence of HIV-1 infection and syphilis in a cohort of police officers in Dar es Salaam, Tanzania: a potential population for HIV vaccine trials. AIDS 2000;14:313-20.

5 Fonck K, Kidula N, Kirui P, et al. Pattern of sexually transmitted diseases and risk factors among women attending an STD referral clinic in Nairobi, Kenya. Sex Transm Dis 2000;27:417-23.

6 Shaw $M$, van der Sande $M$, West $B$, et al. Prevalence of herpes simplex type 2 and syphilis serology among young adults in a rural Gambian community. Sex Transm Infect 2001;77:358-65.

7 Augenbraun MH, DeHovitz JA, Feldman J. Biologic false-positive syphilis test results for women infected with human immunodeficiency virus. Clin Infect Diss 1994; 19:1040-4.

8 Nandwani R, Evans DT. Are you sure it's syphilis? A review of false positive serology. Int J STD AIDS 1995;6:241-8.

9 Erbelding EJ, Vlahov D, Nelson KE, et al. Syphilis serology in human immunodeficiency virus infection: evidence for false-negative fluorescent treponemal testing. J Infect Dis 1997;176:1397-400.

10 Rompalo AM, Cannon RO, Quinn TC, et al. The association of biologic falsepositive serologic tests for syphilis with human immunodeficiency virus infection. J Infect Dis 1992;165:1124-6.

11 Gwanzura L, Latif A, Bassett M, et al. Syphilis serology and HIV infection in Harare, Zimbabwe. Sex Transm Infect 1999;75:426-30.

12 Wicher K, Horowitz HW, Wicher V. Laboratory methods of diagnosis of syphilis for the beginning of the third millenium. Microbes and Infection 1999:1035-49.

13 Young H. Guidelines for serological testing for syphilis. Sex Transm Infect 2000;76:403-5.

14 Young H, Aktas G, Moyes A. Enzywell recombimant enzyme immunoassay for the serological diagnosis of syphilis. Int J STD AIDS 2000:11288-91.

15 World Health Organization. Treponemal infections. Geneva: WHO, Technical Report Series 1982:674.

16 West B, Walraven G, Morison L, et al. Performance of the rapid plasma reagin and the rapid syphilis screening tests in the diagnosis of syphilis in field conditions in rural Africa. Sex Transm Infect 2002;78:282-5.

17 Smikle MF, James OB, Prabhakar P. Biological false positive serological tests for syphilis in the Jamaican population. Genitourin Med 1990;66:76-8.

18 Thomas DL, Quinn TC. Serologic testing for sexually transmitted diseases. Infect Dis Clin N Am 1993:7:793-824.

19 Tsongalis GJ, Berman MM. Application of forensic identity testing in a clinical setting. Specimen identification. Diagnostic Molecular Pathology 1997;6:111-4

20 Suvisaari J, Syrjala M. Practical method for estimating the frequency of specimen mix-up in clinical chemistry laboratories. Clinical Chemistry 2002;48: $1133-5$.

21 Mekonnen Y, Sanders E, Messele T, et al. HIV prevalence, incidence and risk factors for HIV infection among factory workers in Ethiopia. J AIDS 2003 (in press).

22 Nunn AJ, Kengeya-Kayondo JF, Malamba SS, et al. Risk factors for HIV-1 infection in adults in a rural Ugandan community: a population study. AIDS 1994;8:81-6. 